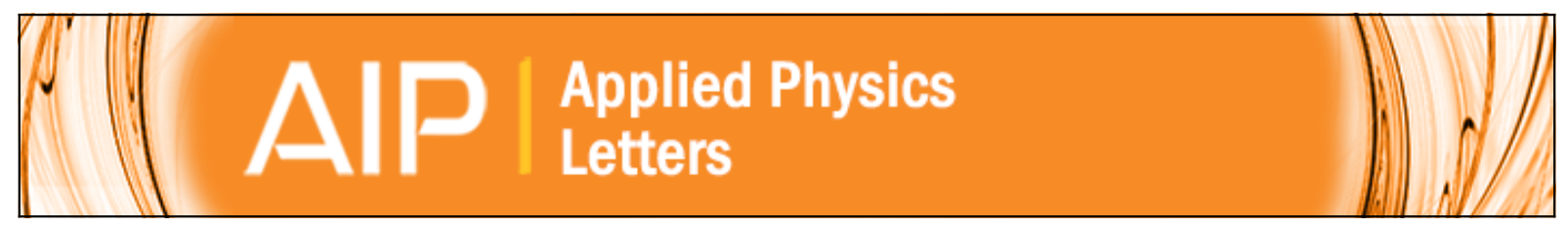

\title{
Synthesis and ferromagnetism of Co-doped Ti 02 nanobelts by metallorganic chemical vapor deposition
}

Nguyen Thi Quynh Hoa, Zonghoon Lee, Seung-Hee Kang, Velimir Radmilovic, and Eui-Tae Kim

Citation: Applied Physics Letters 92, 122508 (2008); doi: 10.1063/1.2904648

View online: http://dx.doi.org/10.1063/1.2904648

View Table of Contents: http://scitation.aip.org/content/aip/journal/apl/92/12?ver=pdfcov

Published by the AIP Publishing

\section{Articles you may be interested in}

Structural and magnetic properties of $\mathrm{Zn} 1 \times \mathrm{Mn} \times \mathrm{O}(0 \times 0.40)$ nanoparticles

J. Appl. Phys. 102, 103907 (2007); 10.1063/1.2815647

Ferromagnetism of Co-doped Ti O 2 ( B ) nanotubes

Appl. Phys. Lett. 91, 143102 (2007); 10.1063/1.2789734

Spin polarization of oxygen atoms in ferromagnetic Co-doped rutile $\mathrm{Ti} O 2$

Appl. Phys. Lett. 89, 182509 (2006); 10.1063/1.2378398

Synthesis and magnetic properties of $\mathrm{Zn} 1 \times \mathrm{Co} \times \mathrm{O}$ nanorods

J. Appl. Phys. 99, 074303 (2006); 10.1063/1.2188031

Semiconducting and ferromagnetic properties of $\mathrm{Ti} 1 \times \mathrm{Co} \times \mathrm{O} 2$ thin films grown by liquid-delivery metalorganic chemical vapor deposition

J. Vac. Sci. Technol. B 22, 762 (2004); 10.1116/1.1688355

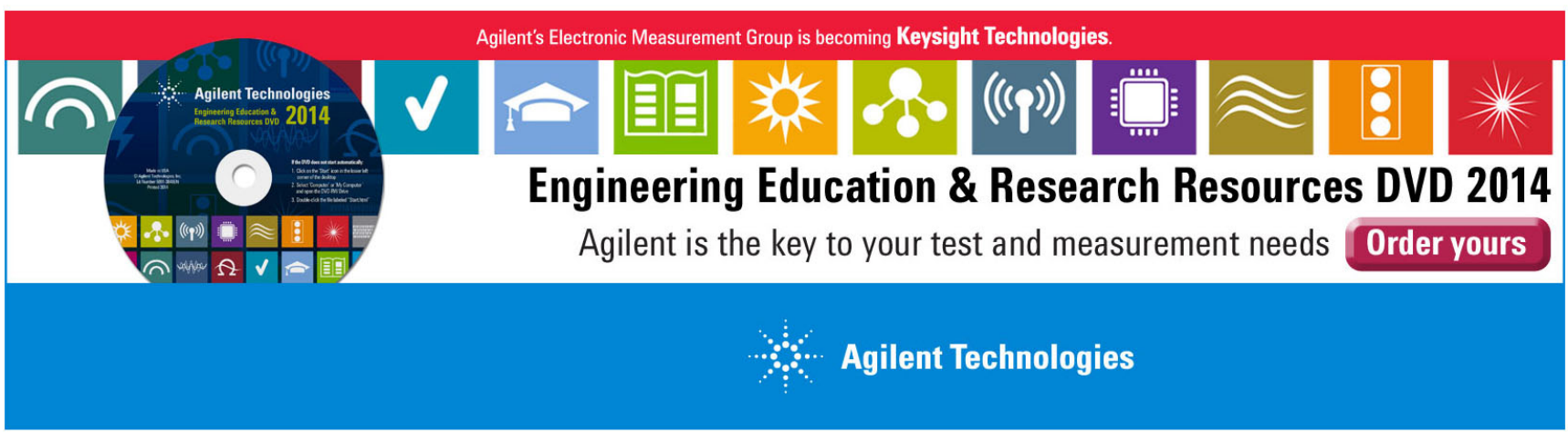




\title{
Synthesis and ferromagnetism of Co-doped $\mathrm{TiO}_{2-\delta}$ nanobelts by metallorganic chemical vapor deposition
}

\author{
Nguyen Thi Quynh Hoa, ${ }^{1}$ Zonghoon Lee, ${ }^{2}$ Seung-Hee Kang, ${ }^{1}$ Velimir Radmilovic, ${ }^{2}$ and \\ Eui-Tae $\mathrm{Kim}^{1, \mathrm{a})}$ \\ ${ }^{1}$ Division of Nano Engineering, Chungnam National University, Daeduk Science Town, \\ Daejeon 305-764, Republic of Korea \\ ${ }^{2}$ National Center for Electron Microscopy, Lawrence Berkeley National Laboratory, 1 Cyclotron Road, MS \\ 72-150, Berkeley, California 94720, USA
}

(Received 28 December 2007; accepted 7 March 2008; published online 28 March 2008)

\begin{abstract}
$\mathrm{Ti}_{1-x} \mathrm{Co}_{x} \mathrm{O}_{2-\delta}$ nanobelts were synthesized without using any metal catalysts by metallorganic chemical vapor deposition. The nanobelts consisted of $\sim 10-20 \mathrm{~nm}$ nanocrystallites, which were dominantly rutile structure. The $\mathrm{Ti}_{0.986} \mathrm{Co}_{0.014} \mathrm{O}_{2-\delta}$ nanobelt sample showed a magnetic anisotropy with a high coercive field value $(\sim 500 \mathrm{Oe})$ at room temperature, significantly affected by intrinsic effect. In contrast, the $\mathrm{Ti}_{0.982} \mathrm{Co}_{0.018} \mathrm{O}_{2-\delta}$ nanobelt sample revealed isotropic magnetic characteristics due to enhanced Co clustering. A few or several Co clusters per nanobelt were typically observed even in the lightly Co-doped $\mathrm{Ti}_{0.986} \mathrm{Co}_{0.014} \mathrm{O}_{2-\delta}$ nanobelts because of the very thin thickness $(\sim 20 \mathrm{~nm})$ and high specific surface area of nanobelts. (C) 2008 American Institute of Physics.

[DOI: $10.1063 / 1.2904648$ ]
\end{abstract}

One-dimensional (1D) diluted magnetic semiconductor (DMS) nanostructures are of intense interest for fundamental research and promising spintronic device applications. ${ }^{1-3}$ DMS materials with the Curie temperature $T_{C}$ beyond room temperature (RT) have been extensively studied in realizing the integration of practical spintronic and electronic devices. $^{4-10}$ Among potential DMS materials, transition metal-doped $\mathrm{TiO}_{2}$ has been extensively studied as one of the most promising candidates ${ }^{5-10}$ since cobalt $(\mathrm{Co})$-doped $\mathrm{TiO}_{2}$ $\left(\mathrm{Ti}_{1-x} \mathrm{Co}_{x} \mathrm{O}_{2}\right)$ was shown to be ferromagnetic with $T_{C}$ up to $400 \mathrm{~K} .{ }^{5}$ However, little information is available on the synthesis and characteristics of $\mathrm{Ti}_{1-x} \mathrm{Co}_{x} \mathrm{O}_{2} 1 \mathrm{D}$ nanostructures. Synthesis methods of $\mathrm{Ti}_{1-x} \mathrm{Co}_{x} \mathrm{O}_{2}$ 1D nanostructures have been limited to template-assistant fabrication ${ }^{2,3}$ and solutionbased techniques such as hydrothermal treatment ${ }^{1}$ so far. Synthesis of $\mathrm{Ti}_{1-x} \mathrm{Co}_{x} \mathrm{O}_{2}$ 1D nanostructures by chemical vapor deposition (CVD) represents a challenging issue which has not been reported thus far. CVD has several advantages over other reported approaches for compatibility with silicon technology and mass production on large-scale wafers. Another key issue is the origin of the RT ferromagnetism (RTFM) of $\mathrm{Ti}_{1-x} \mathrm{Co}_{x} \mathrm{O}_{2}$, whether it is due to an intrinsic or an extrinsic effect (Co clusters). ${ }^{5-10}$ Most of these studies have been focused on thin films, but very few investigations of $\mathrm{Ti}_{1-x} \mathrm{Co}_{x} \mathrm{O}_{2}$ 1D nanostructures have been reported. Moreover, the Co concentrations of the studied $\mathrm{Ti}_{1-x} \mathrm{Co}_{x} \mathrm{O}_{2}$ nanotubes were more than 4 at. \%. ${ }^{1,2}$ Such a Co concentration can be high enough to form Co clusters. Co clustering has been reported in rutile $\mathrm{Ti}_{0.98} \mathrm{Co}_{0.02} \mathrm{O}_{2-\delta}$ thin films. ${ }^{8}$ Our recent study also shows that the solubility of $\mathrm{Co}$ in anatase $\mathrm{Ti}_{1-x} \mathrm{Co}_{x} \mathrm{O}_{2-\delta}$ thin films was significantly low $(<3$ at. $\%){ }^{9}$ The high specific surface area of 1D nanostructures can enhance Co clustering because Co atoms strongly tend to segregate onto the surface. In this letter, we report the selfcatalytic growth of $\mathrm{Ti}_{1-x} \mathrm{Co}_{x} \mathrm{O}_{2-\delta}$ nanobelts via metallorganic

${ }^{\text {a) }}$ Author to whom correspondence should be addressed. Electronic mail: etkim@cnu.ac.kr.
CVD (MOCVD) without using any metal catalysts. The Co clustering and RTFM characteristics of very lightly Codoped $\mathrm{Ti}_{1-x} \mathrm{Co}_{x} \mathrm{O}_{2-\delta}(x<2$ at. \%) nanobelts have been thoroughly investigated.

$\mathrm{Ti}_{1-x} \mathrm{Co}_{x} \mathrm{O}_{2-\delta}$ nanobelts were grown on bare sapphire (0001) substrate by MOCVD using $\left(\mathrm{C}_{11} \mathrm{H}_{19} \mathrm{O}_{2}\right)_{2}\left(\mathrm{C}_{3} \mathrm{H}_{7} \mathrm{O}\right)_{2} \mathrm{Ti}$ and $\mathrm{Co}\left(\mathrm{C}_{11} \mathrm{H}_{19} \mathrm{O}_{2}\right)_{3}$. The growth temperature and the pressure were fixed at $510{ }^{\circ} \mathrm{C}$ and 10 Torr, respectively. The Ti and the Co precursors were bubbled with an argon (Ar) gas flow of 150 and 50 SCCM (SCCM denotes cubic centimeter per minute at STP), respectively. The total flow rate was fixed at $300 \mathrm{SCCM}$. We noted that all $\mathrm{Ti}_{1-x} \mathrm{Co}_{x} \mathrm{O}_{2-\delta}$ nanobelt samples studied here were grown by supplying Ar gas only. As a result, the $\mathrm{Ti}_{1-x} \mathrm{Co}_{x} \mathrm{O}_{2-\delta}$ nanobelt samples were highly reduced and showed a very low resistivity (a few $\Omega \mathrm{cm}$ ). The bubbling temperature of the $\mathrm{Ti}$ precursor was fixed at $200{ }^{\circ} \mathrm{C}$. The Co doping concentration could be controlled by varying Co bubbling temperature. The slope $(-\Delta H)$ of the Clausius-Clapeyron equation was gentle enough that Co vapor pressure could be controlled reliably for such a low doping amount used here. The average Co doping concentrations were found to be 1.4 and 1.8 at. \% for 180 and $185{ }^{\circ} \mathrm{C}$ of the Co bubbling temperatures, respectively, as analyzed by an electron probe microanalyzer. $\mathrm{The}^{\mathrm{Ti}_{1-x}} \mathrm{Co}_{x} \mathrm{O}_{2-\delta}$ nanobelts were characterized by vibrating sample magnetometer (Lake Shore 7400 series), field-emission scanning electron microscopy (SEM) (Jeol JSM 7000F), transmission electron microscopy (TEM), and photoluminescence (PL) spectroscopy. To investigate Co clustering, we carried out high angle annular dark field (HADDF) scanning TEM (STEM) study using FEI Tecnai F20 UT.

Figure 1 is a SEM image of $\mathrm{Ti}_{0.986} \mathrm{Co}_{0.014} \mathrm{O}_{2-\delta}$ nanobelts grown on a sapphire (0001) substrate for $10 \mathrm{~min}$. The image shows that nanobelts were formed at a high density and a bunch of several nanobelts were branched at the same place. The nanobelts are $\sim 2 \mu \mathrm{m}$ in length and the width and the thickness are $\sim 100-150$ and $\sim 20 \mathrm{~nm}$, respectively. Figure 2(a) shows a typical bright-field TEM image of a single 


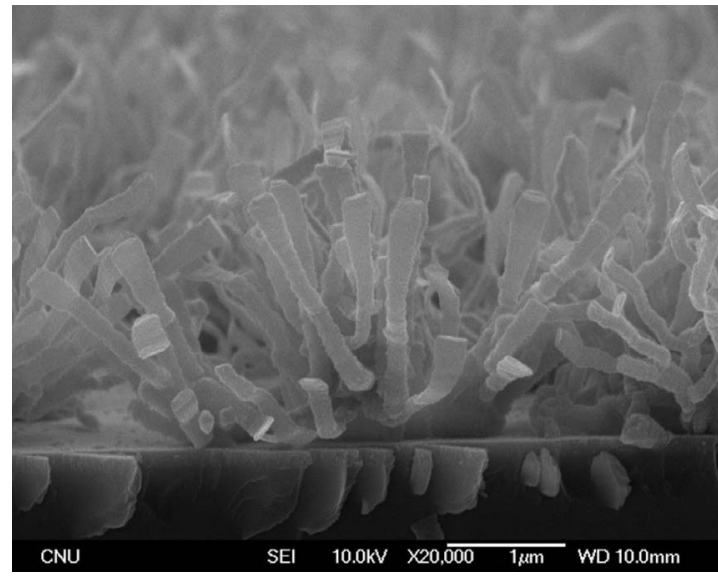

FIG. 1. SEM image of $\mathrm{Ti}_{0.986} \mathrm{Co}_{0.014} \mathrm{O}_{2-\delta}$ nanobelts on sapphire substrate.

$\mathrm{Ti}_{0.986} \mathrm{Co}_{0.014} \mathrm{O}_{2-\delta}$ nanobelt. The TEM image reveals that nanobelts consisted of $\sim 10-20 \mathrm{~nm}$ size nanocrystallites. Moreover, the high-resolution lattice image [Fig. 2(b)] shows that the nanocrystallites are well aligned with one another. The inset of Fig. 2(b) shows the corresponding digital diffractogram pattern, indicating that nanocrystallites of nanobelts are oriented along the [211] zone-axis rutile phase.

The magnetic properties of the $\mathrm{Ti}_{1-x} \mathrm{Co}_{x} \mathrm{O}_{2-\delta}$ nanobelt samples were investigated with the magnetic field along in plane $\left(H_{\|}\right)$and out of plane $\left(H_{\perp}\right)$ at RT. The diamagnetic background of the substrate was subtracted from the data. As seen in Fig. 3, the $\mathrm{Ti}_{0.986} \mathrm{Co}_{0.014} \mathrm{O}_{2-\delta}$ nanobelt sample reveals the magnetization hysteresis loops with the coercive field $\left(H_{C}\right)$ of $\sim 500 \mathrm{Oe}$, indicating RTFM. Moreover, the sample shows anisotropic magnetic characteristics that the saturation magnetization $\left(M_{S}\right)$ value $(\sim 40 \mathrm{memu} / \mathrm{g})$ at $H_{\|}$is slightly larger than that $(\sim 30 \mathrm{memu} / \mathrm{g})$ at $H_{\perp}$. The anisotropic magnetic characteristics may indicate the possibility of the intrinsic DMS characteristics. It was reported that FM by Co clusters in $\mathrm{Ti}_{1-x} \mathrm{Co}_{x} \mathrm{O}_{2-\delta}$ thin films did not show any significant magnetic anisotropy. ${ }^{7}$ With increased Co doping level to 1.8 at. \%, isotropic magnetic characteristics with a significantly increased $M_{S}$ value $(\sim 200 \mathrm{memu} / \mathrm{g})$ were observed (the inset in Fig. 3).

To further investigate the origin of the RTFM characteristics of $\mathrm{Ti}_{1-x} \mathrm{Co}_{x} \mathrm{O}_{2-\delta}$ nanobelt samples, HADDF STEM study was carried out. Figure 4 shows a typical HADDF STEM image of a single nanobelt of the $\mathrm{Ti}_{0.986} \mathrm{Co}_{0.014} \mathrm{O}_{2-\delta}$ sample. The bright spots in the nanobelt tip region reveal the high concentration of Co element, indicating Co clusters
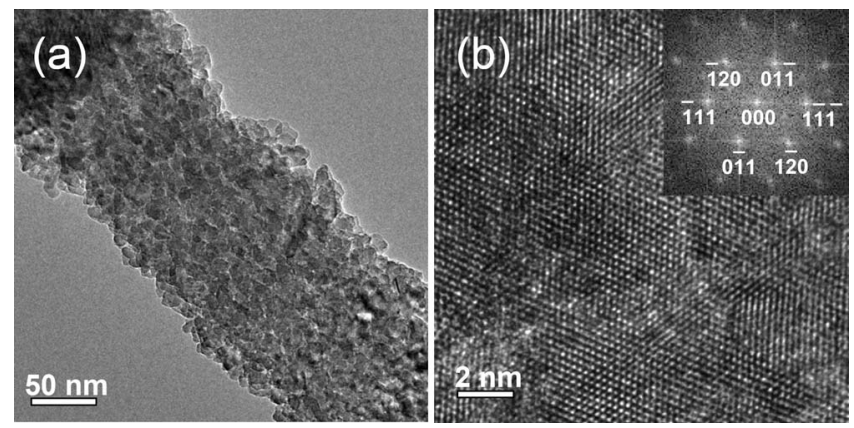

FIG. 2. Typical TEM images of a $\mathrm{Ti}_{0.986} \mathrm{Co}_{0.014} \mathrm{O}_{2-\delta}$ nanobelt. (a) A lowmagnification TEM image. (b) A HRTEM image and its digital diffractogram pattern (inset).

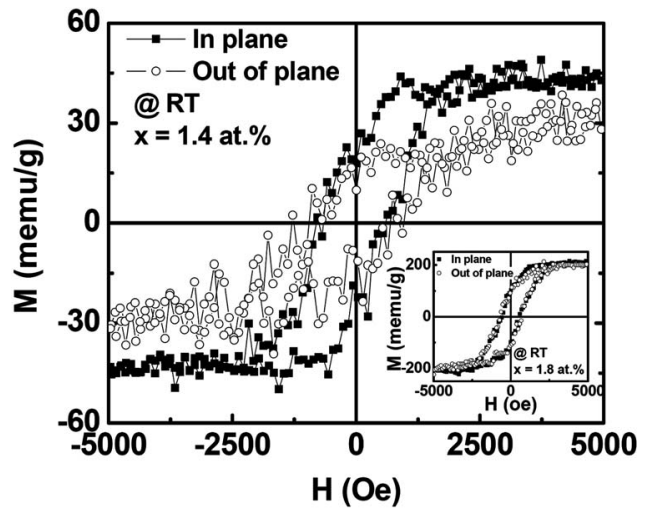

FIG. 3. Magnetization curves of the $\mathrm{Ti}_{0.986} \mathrm{Co}_{0.014} \mathrm{O}_{2-\delta}$ and $\mathrm{Ti}_{0.986} \mathrm{Co}_{0.014} \mathrm{O}_{2-\delta}$ nanobelt samples with the magnetic field along in plane and out of plane at RT.

$(\sim 10 \mathrm{~nm}$ size $) .{ }^{9}$ A few or several Co clusters per nanobelt were typically observed. Nevertheless, the possibility of intrinsic RTFM cannot be completely ruled out. The magnetic anisotropy with the high $H_{C}$ value $(\sim 500 \mathrm{Oe})$ cannot be explained by the small number of $\sim 10 \mathrm{~nm}$ size Co clusters. Shinde et $^{\mathrm{al}}{ }^{8}$ reported that $\sim 8-10 \mathrm{~nm}$ Co clusters in rutile $\mathrm{Ti}_{0.98} \mathrm{Co}_{0.02} \mathrm{O}_{2-\delta}$ thin film showed very small $H_{C}(>50 \mathrm{Oe})$ at RT. In contrast, the isotropic magnetic characteristics of the $\mathrm{Ti}_{0.982} \mathrm{Co}_{0.018} \mathrm{O}_{2-\delta}$ nanobelt sample seem to be dominantly affected by Co clusters (the inset of Fig. 3). Co clustering occurred at a lower Co doping concentration $(\sim 1.4$ at. \%) than previously reported results. ${ }^{7-10}$ We note that the grown $\mathrm{Ti}_{1-x} \mathrm{Co}_{x} \mathrm{O}_{2-\delta}$ nanobelts are as thin as just $\sim 20 \mathrm{~nm}$. Co atoms have a strong tendency to segregate and form clusters on the surface of $\mathrm{Ti}_{1-x} \mathrm{Co}_{x} \mathrm{O}_{2-\delta}$ during CVD growth ${ }^{9}$ and annealing process. ${ }^{10}$ Compared to bulk or thin film, Co clustering can be significantly enhanced in the nanobelts because of a very thin thickness $(\sim 20 \mathrm{~nm})$ and high specific surface area. Moreover, oxygen vacancies in nanobelts and oxygen-poor CVD growth conditions can stimulate Co clustering further.

Figure 5 shows the PL spectra of undoped $\mathrm{TiO}_{2-\delta}$, $\mathrm{Ti}_{0.986} \mathrm{Co}_{0.014} \mathrm{O}_{2-\delta}$, and $\mathrm{Ti}_{0.982} \mathrm{Co}_{0.018} \mathrm{O}_{2-\delta}$ nanobelt samples at $4 \mathrm{~K}$. The nanobelt samples yield a strong broad PL peak centered at $\sim 530 \mathrm{~nm}$ along with a very weak peak at $\sim 400 \mathrm{~nm}$. The PL peak at $\sim 400 \mathrm{~nm}$ is assigned to the recombination of excitons from the band edge of $\mathrm{TiO}_{2}$. The visible-light peak at $\sim 530 \mathrm{~nm}$ can be attributed to the recombination from various defects such as oxygen vacancy and $\mathrm{Ti}^{3+}$. By increasing the Co doping concentration, the PL
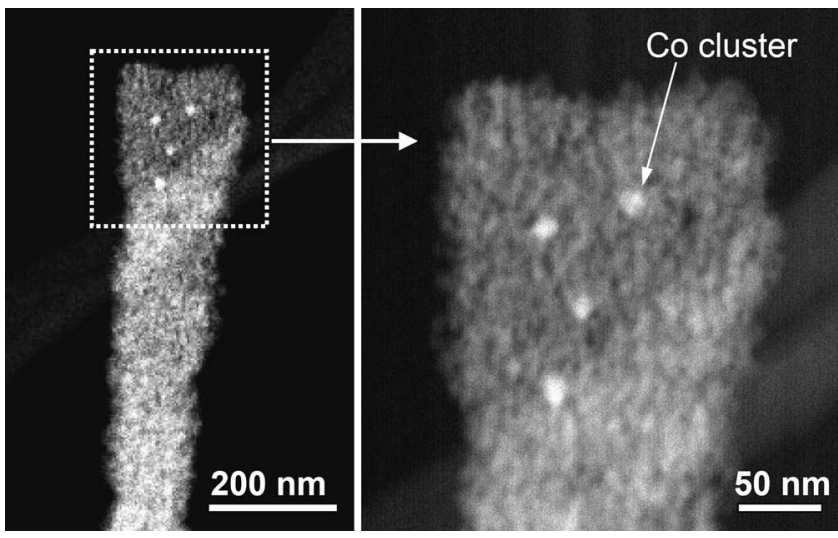

FIG. 4. HADDF STEM image of a $\mathrm{Ti}_{0.986} \mathrm{Co}_{0.014} \mathrm{O}_{2-\delta}$ nanobelt. 


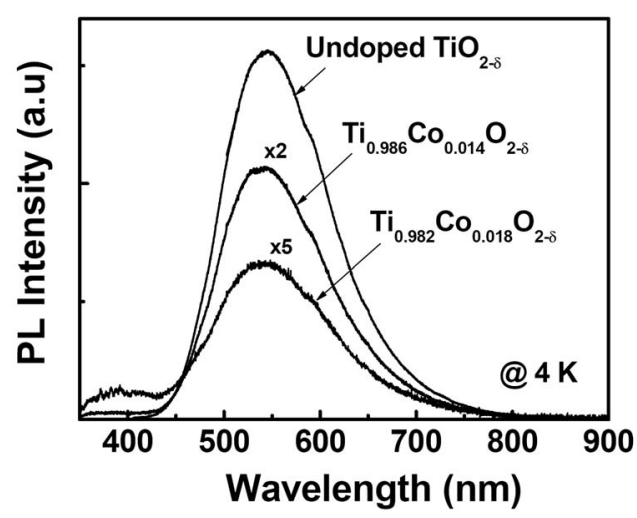

FIG. 5. PL spectra of undoped $\mathrm{TiO}_{2-\delta}, \mathrm{Ti}_{0.986} \mathrm{Co}_{0.014} \mathrm{O}_{2-\delta}$, and $\mathrm{Ti}_{0.982} \mathrm{Co}_{0.018} \mathrm{O}_{2-\delta}$ nanobelt samples at $4 \mathrm{~K}$.

intensity remarkably decreases. It indicates that Co dopants act as nonradiative recombination sites and are effectively distributed to the $\mathrm{TiO}_{2-\delta}$ matrix. We note that the PL peak position of the $\mathrm{Ti}_{0.986} \mathrm{Co}_{0.014} \mathrm{O}_{2-\delta}$ nanobelt sample is slightly blueshifted $(\sim 20 \mathrm{meV})$ with respect to that of the undoped $\mathrm{TiO}_{2-\delta}$ nanobelt sample. Meanwhile, the PL peak positions of $\mathrm{Ti}_{0.986} \mathrm{Co}_{0.014} \mathrm{O}_{2-\delta}$ and $\mathrm{Ti}_{0.982} \mathrm{Co}_{0.018} \mathrm{O}_{2-\delta}$ nanobelt samples are almost identical. Guha et al. ${ }^{11}$ reported that the PL peak at $\sim 540 \mathrm{~nm}$ was blueshifted with increased Co doping amount in epitaxial anatase $\mathrm{Ti}_{1-x} \mathrm{Co}_{x} \mathrm{O}_{2}$ thin films. The PL peak position was not significantly changed above $\sim 2$ at. $\%$, that they thought was limited Co solubility. In this study, however, the PL peak position is not further blueshifted with increasing Co doping amount from 1.4 to 1.8 at. \%. It may indicate that further doped $\mathrm{Co}$ atoms are segregated for $\mathrm{Co}$ clusters, which contributes to the observed isotropic magnetic characteristics. With increasing temperature, the PL intensity of the $\mathrm{Ti}_{0.986} \mathrm{Co}_{0.014} \mathrm{O}_{2-\delta}$ nanobelt sample was not significantly changed in the range of $4-50 \mathrm{~K}$. Above $50 \mathrm{~K}$, the PL intensity was exponentially decreased with the activation energy of $43 \pm 5 \mathrm{meV}$, which is attributed to losing holes and electrons into thermally activated nonradiative channels. A very week PL spectrum centered at $\sim 500 \mathrm{~nm}$ was still observed at RT.

Finally, we like to mention that a vapor-solid (VS) growth mechanism excluding a liquid phase appears to control the $\mathrm{Ti}_{1-x} \mathrm{Co}_{x} \mathrm{O}_{2-\delta}$ nanobelt formation. ${ }^{12}$ It should be emphasized that $\sim 100 \mathrm{~nm}$ thick thin film was first grown in the earlier growth stage before the formation of the nanobelts. The thin film can provide thermodynamically stable crystal structures acting as the seed for the nanobelt growth. ${ }^{12,13}$ It has been reported that predeposited thin film was essential for the ensuing self-catalytic growth of nanowires. ${ }^{12,13}$ More details of the self-catalytic growth of $\mathrm{TiO}_{2-\delta}$ nanobelts by MOCVD were reported elsewhere. ${ }^{12}$

In conclusion, Co-doped $\mathrm{TiO}_{2-\delta}$ nanobelts were grown via a VS mechanism without the use of any metal catalysts using MOCVD. The nanobelts consisted of $\sim 10-20 \mathrm{~nm}$ nanocrystallites, which were dominantly rutile structures and well-aligned to one another. An intrinsic effect seemed to significantly contribute to the anisotropic RTFM of the $\mathrm{Ti}_{0.986} \mathrm{Co}_{0.014} \mathrm{O}_{2-\delta}$ nanobelt sample even though a few $\sim 10 \mathrm{~nm}$ Co clusters per nanobelt were observed. By increasing the average Co doping concentration to 1.8 at. $\%$, the nanobelt sample revealed isotropic magnetic characteristics due to enhanced Co clustering. Co clustering could not be suppressed even in such a lightly doped $\mathrm{Ti}_{1-x} \mathrm{Co}_{x} \mathrm{O}_{2}$ $(x \sim 1.4$ at. \% $)$ nanobelts because of the very thin thickness $(\sim 20 \mathrm{~nm})$ and high specific surface area of nanobelts.

This work is supported by the Korea Research Foundation Grant funded by the Korean Government (MOEHRD) (KRF-2006-331-D00260). The authors acknowledge support of the National Center for Electron Microscopy, Lawrence Berkeley Lab, which is supported by the U.S. Department of Energy under Contract No. DE-AC02-05CH11231.

${ }^{1}$ D. Wu, Y. Chen, J. Liu, X. Zhao, A. Li, and N. Ming, Appl. Phys. Lett. 87, 112501 (2005).

${ }^{2}$ C. Huang, X. Liu, Y. Liu, and Y. Wang, Chem. Phys. Lett. 432, 468 (2006).

${ }^{3}$ Y. H. Lee, J. M. Yoo, D. H. Park, D. H. Kim, and B. K. Ju, Appl. Phys. Lett. 86, 033110 (2005).

${ }^{4}$ H. Ohno, Science 281, 951 (1998).

${ }^{5}$ Y. Matsumoto, M. Murakami, T. Shono, T. Hasegawa, T. Fukumura, M. Kawasaki, P. Ahmet, T. Chikyow, S.-Y. Koshihara, and H. Koninuma, Science 291, 854 (2001).

${ }^{6}$ K. A. Griffin, A. B. Pakhomov, C. M. Wang, S. M. Heald, and K. M. Krishnan, Phys. Rev. Lett. 94, 157204 (2005).

${ }^{7}$ J.-Y. Kim, J.-H. Park, B.-G. Park, H.-J. Noh, S.-J. Oh, J. S. Yang, D.-H. Kim, S. D. Bu, T.-W. Noh, H.-J. Lin, H.-H. Hsieh, and C. T. Chen, Phys. Rev. Lett. 90, 017401 (2003).

${ }^{8}$ S. R. Shinde, S. B. Ogale, J. S. Higgins, H. Zheng, A. J. Millis, V. N. Kulkarni, R. Ramesh, R. L. Greene, and T. Venkatesan, Phys. Rev. Lett. 92, 166601 (2004).

${ }^{9}$ S. H. Kang, H. N. T. Quynh, S. G. Yoon, E. T. Kim, Z. Lee, and V. Radmilovic, Appl. Phys. Lett. 90, 102504 (2007).

${ }^{10}$ N. J. Seong, S. G. Yoon, and C. R. Cho, Appl. Phys. Lett. 81, 4209 (2002).

${ }^{11}$ S. Guha, K. Ghosh, J. G. Keeth, S. B. Ogale, S. R. Shinde, J. R. Simpson, H. D. Drew, and T. Venkatesan, Appl. Phys. Lett. 83, 3296 (2003).

${ }^{12}$ N. T. Q. Hoa and E. T. Kim, Electrochem. Solid-State Lett. 11, K1 (2008).

${ }^{13}$ J. M. Wu, H. C. Shih, and W. T. Wu, Nanotechnology 17, 105 (2006). 